\title{
Preoperative risk score and in-hospital death following isolated myocardial
}

\section{revascularization surgery}

\author{
Avaliação do risco de óbito intra-hospitalar em cirurgia de revascularização miocárdia isolada
}

\author{
através do ERPO
}

Evaluación del riesgo de muerte intrahospitalaria en cirugía de revascularización miocárdica aislada mediante ERPO

Received: 01/17/2022 | Reviewed: 01/25/2022 | Accept: 01/25/2022 | Published: 01/26/2022

\author{
Raphael Paris Rosan \\ ORCID: https://orcid.org/0000-0002-0107-7726 \\ Dante Pazzanese Institute of Cardiology, Brazil \\ E-mail: RaphaelParis7@hotmail.com \\ Pedro Silvio Farsky \\ ORCID: https://orcid.org/0000-0001-7427-3237 \\ Dante Pazzanese Institute of Cardiology, Brazil \\ E-mail: pedro.farsky@gmail.com \\ João Ítalo Dias França \\ ORCID: https://orcid.org/0000-0001-5606-5960 \\ Dante Pazzanese Institute of Cardiology, Brazil \\ E-mail: jitalo@lee.dante.br \\ Vivian Lerner Amato \\ ORCID: https://orcid.org/0000-0003-1512-3444 \\ Dante Pazzanese Institute of Cardiology, Brazil \\ E-mail: vlerner@cardiol.br
}

\begin{abstract}
Cardiovascular disease is the leading cause of death in Brazil. The risk of in-hospital death after coronary artery bypass grafting $(\mathrm{CABG})$ can be assessed by identifying preoperative factors and quantified through scores. This investigation analyzed associated with mortality in the hospital phase after isolated CABG, to develop a preoperative risk score (PRS) for postoperative in-hospital death. This observational, retrospective, single-center study examined comorbidities and complementary exams of 9,826 patients who underwent isolated CABG between January 1, 1999 and December 31, 2017 were analyzed to identify their correlation with postoperative in-hospital death. A total of 9,826 patients were divided into the construction group $(7,860 ; 80 \%)$ and validation group $(1,966 ; 20 \%)$. The mean age of the patients was 62.43 years. Most patients were men (70.2\%). Fifteen independently related factors were identified for hospital mortality. The final logistic model was calculated in the construction group with a C-statistic of 0.745 (95\% confidence interval [CI], 0.720-0.770). In the validation group, the score was tested by obtaining an area under the receiver operating curve of 0.716 (95\% [CI], 0.666-0.767). Death occurred in 489 patients (5\%). The risk of death ranged from $1.2 \%$ in those considered to be low risk (PRS <85) to $16.3 \%$ among those considered to be very high risk (PRS >211). The PRS score satisfactorily discriminated among patients who exhibited low, intermediate, high, and very high risk of death in the postoperative period. PRS could serve as an auxiliary tool in the preoperative period for surgical teams.
\end{abstract}

Keywords: Cardiovascular disease; Coronary artery bypass grafting; Preoperative risk score.

\section{Resumo}

A doença cardiovascular é a principal causa de morte no Brasil. O risco de óbito intra-hospitalar após cirurgia de revascularização do miocárdio (RM) pode ser avaliado pela identificação de fatores pré-operatórios e quantificado por meio de escores. Esta investigação analisou a mortalidade na fase hospitalar após CRM isolada, para desenvolver um escore de risco pré-operatório (PRS) para óbito intra-hospitalar pós-operatório. Este estudo observacional, retrospectivo, unicêntrico examinou comorbidades e exames complementares de 9.826 pacientes 
submetidos à CRM isolada entre $1^{\circ}$ de janeiro de 1999 e 31 de dezembro de 2017 e foram analisados para identificar sua correlação com óbito intra-hospitalar pós-operatório. Um total de 9.826 pacientes foi dividido em grupo de construção $(7.860 ; 80 \%)$ e grupo de validação $(1.966 ; 20 \%)$. A média de idade dos pacientes foi de 62,43 anos sendo a maioria homens $(70,2 \%)$. Quinze fatores independentemente relacionados foram identificados para a mortalidade hospitalar. O modelo logístico final foi calculado no grupo de construção com uma estatística C de 0,745 (intervalo de confiança de $95 \%$ [IC], 0,720-0,770). No grupo de validação, o escore foi testado obtendo-se uma área sob a curva operacional do receptor de 0,716 (95\% [IC], 0,666-0,767). A morte ocorreu em 489 pacientes $(5 \%)$. O risco de óbito variou de $1,2 \%$ nos considerados de baixo risco (PRS <85) a 16,3\% nos considerados de altíssimo risco (PRS >211). O escore PRS discriminou satisfatoriamente os pacientes que apresentaram risco baixo, intermediário, alto e muito alto de óbito no pós-operatório, podendo servir como ferramenta auxiliar no pré-operatório para as equipes cirúrgicas.

Palavras-chave: Doença cardiovascular; Cirurgia de revascularização do miocárdio; Escore de risco préoperatório.

\section{Resumen}

La enfermedad cardiovascular es la principal causa de muerte en Brasil. El riesgo de muerte en el hospital después de un injerto de derivación de la arteria coronaria (CABG) se puede evaluar mediante la identificación de factores preoperatorios y cuantificar a través de puntuaciones. Esta investigación analizó la mortalidad asociada a la fase hospitalaria después de una CABG aislada, para desarrollar una puntuación de riesgo preoperatorio (PRS) para la muerte intrahospitalaria posoperatoria. Este estudio observacional, retrospectivo, unicéntrico, examinó las comorbilidades y los exámenes complementarios de 9826 pacientes que se sometieron a CABG aislada entre el 1 de enero de 1999 y el 31 de diciembre de 2017 para identificar su correlación con la muerte hospitalaria postoperatoria. Un total de 9826 pacientes se dividieron en el grupo de construcción (7860; 80\%) y el grupo de validación (1966; 20\%). La edad media de los pacientes fue de 62,43 años. La mayoría de los pacientes eran hombres $(70,2 \%)$. Se identificaron quince factores relacionados de forma independiente para la mortalidad hospitalaria. El modelo logístico final se calculó en el grupo de construcción con una estadística $\mathrm{C}$ de 0,745 (intervalo de confianza [IC] del $95 \%, 0,720-0,770)$. En el grupo de validación, se probó la puntuación obteniendo un área bajo la curva operativa del receptor de 0,716 (95\% [IC], 0,666-0,767). La muerte ocurrió en 489 pacientes (5\%). El riesgo de muerte varió del 1,2\% en los considerados de bajo riesgo (PRS <85) al 16,3\% en los considerados de muy alto riesgo (PRS > 211). La puntuación PRS discriminó satisfactoriamente entre los pacientes que presentaban un riesgo de muerte en el postoperatorio bajo, intermedio, alto y muy alto. La PRS podría servir como herramienta auxiliar en el preoperatorio de los equipos quirúrgicos.

Palabras clave: Enfermedad cardiovascular; Bypass aortocoronario; Score de riesgo preoperatorio.

\section{Introduction}

Cardiovascular disease is the leading cause of death in Brazil. According to Health Ministry data from DATASUS, diseases of the circulatory system were the main cause of death, accounting for 6,283,717 deaths, from 1996 to 2016 (Health Ministery, 2021). Data analysis from 2016 shows that coronary atherosclerotic disease (CAD) was responsible for $29.7 \%$ of the 362,091 deaths associated with cardiovascular disease (Health Ministery, 2021).

Myocardial revascularization (percutaneous or surgical) is an integral part of a global CAD treatment strategy. Risk scores are available for coronary artery bypass grafting (CABG) and cardiac surgery, in general, making it possible to calculate the risk of postoperative mortality, stroke, acute myocardial infarction (AMI), and renal failure (Nilsson et al., 2006).

Along with the Society of Thoracic Surgeons (STS) and EuroSCORE II scores (the most commonly used scores in practice), additional scores can be used to estimate hospital mortality in CABG (D'Agostino et al., 2019; Nashef et al., 2012). However, it is essential to identify the factors associated with deaths in Brazil and build the most appropriate scoring system for this population at the national level. 
Research, Society and Development, v. 11, n. 2, e34211225828, 2022

(CC BY 4.0) | ISSN 2525-3409 | DOI: http://dx.doi.org/10.33448/rsd-v11i2.25828

This study aimed to analyze the elements associated with mortality in the hospital phase after isolated CABG and to develop a preoperative risk score (PRS).

\section{Patients And Methods}

\subsection{Study design}

This observational, retrospective, single-center study was conducted by the Dante Pazzanese Institute of Cardiology (IDPC). The IDPC ethics and research committee approved the research in March 15, 2019 under the number: 3.201.428. The requirement for informed consent was waived because it was a retrospective study.

\subsection{Population}

Patients who underwent isolated CABG surgery at IDPC from January 1, 1999 to December 31, 2017 and whose records appear in the database of the hospital sector of coronary diseases at IDPC were included.

Patients who underwent other procedures associated with CABG, such as valve approach (repair or replacement), carotid endarterectomy, left ventricular aneurysmectomy, or an aortic aneurysm approach, were excluded.

\subsection{Study procedures}

First, we analyzed the preoperative factors associated with mortality in the hospital phase in patients undergoing isolated CABG. Second, we analyzed the identified factors and quantified the correlation with death outcomes in the hospital phase. Finally, we developed a risk score for hospital mortality to be used preoperatively in similar populations.

\subsection{Analyzed variables}

The analyzed variables (Table 1) were personal data, medical history, clinical diagnosis, angiographic results, and surgical procedure. The outcomes included hospital discharge or death. 
Table 1. Analyzed characteristics of patients.

\begin{tabular}{ll}
\hline \multicolumn{1}{c}{ Patient information } & \multicolumn{1}{c}{ Analyzed characteristics } \\
\hline Personal Data & Age, Sex, Race/Ethnicity, Weight, BSA, BMI \\
Medical history & SAH, DM, Dyslipidemia, Family history of early CAD, Current or past smoking, COPD, CKD, PNE \\
& (TIA+ stroke), Carotid artery disease, PAD, Previous AMI \\
& Stable angina, unstable angina, silent ischemia, asymptomatic after AMI \\
Clinical diagnosis & $\begin{array}{l}\text { Presence of angina and its class according to CCS } \\
\text { Presence of dyspnea and its class according to NYHA }\end{array}$ \\
Coronary Angiogram / & Affected vessels, \\
ventriculography & Significant injury of LMD, LVEF \\
Surgical procedure & Elective, Urgent, Emergent \\
Use of cardiopulmonary bypass \\
Grafts performed \\
Length of hospital stay after surgery \\
Hospital discharge or Death \\
\hline
\end{tabular}

BSA, body surface area; BMI, body mass index; SAH, systemic arterial hypertension; DM, diabetes mellitus; CAD, coronary atherosclerotic disease; COPD, chronic obstructive pulmonary disease; CKD, chronic kidney disease; PNE, previous neurological event; TIA, transient ischemic attack; PAD, peripheral arterial disease; AMI, acute myocardial infarction; CCS, Canadian Cardiovascular Society; NYHA, New York Heart Association; LMD, left main disease; LVEF, left ventricular ejection fraction. Source: Authors.

\subsection{Statistical analyses}

A multivariate approach was used, using the logistic regression technique with the stepwise method for selecting variables, to assess the influence of risk factors. Hospital death was considered a dependent variable. The results for this analysis were expressed as odds ratios with respective 95\% confidence intervals (CIs).

The variables introduced in the multivariate analysis model were selected based on the results of the univariate analysis with $\mathrm{P}<0.15$ and previous knowledge of the factors associated with death, namely: age, sex, hypertension, diabetes, a previous neurological event (PNE) (transient ischemic attack or stroke), creatinine level $>1.5 \mathrm{mg} / \mathrm{dL}$, carotid obstruction $>50 \%$, peripheral arterial disease (PAD), previous AMI, left ventricular ejection fraction (LVEF), dyspnea class according to the New York Heart Association (NYHA), angina class according to Canadian Cardiovascular Society (CCS), surgical indication (elective, urgent, or emergent), left main disease (LMD) $>50 \%$, and current smoker. The C-statistic was calculated to measure the predictive power of the model.

To develop PRS, all patients were included in a random list. Through the R Core program, $80 \%$ were allocated to the construction group and $20 \%$ to the validation group.

PRS estimated the chance of death in the in-hospital phase of the CABG postoperative period, with higher scores indicating greater risk. $\mathrm{P} \leq 0.05$ was considered significant. 


\section{Results}

Table 2 shows patients divided into the construction group, $(7,860 ; 80 \%)$ and the validation group, $(1,966 ; 20 \%)$. Both groups had similar characteristics, except for the incidence of diabetes mellitus (42.3\% in the construction group vs. $40.0 \%$ in the validation group; $\mathrm{P}=0.02$ ).

Table 2. Baseline characteristics of the construction and validation groups.

\begin{tabular}{|c|c|c|c|c|}
\hline Characteristic & $\begin{array}{l}\text { Total } \\
(\mathrm{N}=\mathbf{9 , 8 2 6})\end{array}$ & $\begin{array}{l}\text { Construction } \\
(\mathrm{N}=\mathbf{7 , 8 6 0})\end{array}$ & $\begin{array}{l}\text { Validation } \\
(\mathrm{N}=1,966)\end{array}$ & $\overline{\text { P-Value }}$ \\
\hline Age in years - no./total no. (\%) & & & & 0.215 \\
\hline$\leq \mathbf{5 0}$ & $1,139 / 9,814(11.6)$ & $890 / 7,850(11.3)$ & 249/1,964 (12.7) & \\
\hline $50-69$ & $6,247 / 9,814(63.7)$ & $5,003 / 7,850(63.7)$ & $1,244 / 1,964(63.3)$ & \\
\hline$\geq 70$ & $2,428 / 9,814(24.7)$ & $1,957 / 7,850(24.7)$ & 471/1,964 (24.0) & \\
\hline Sex - no./total no. (\%) & & & & 0.393 \\
\hline Male & $6,895 / 9,826(70.2)$ & $5,531 / 7,860(70.4)$ & $1,364 / 1,966(69.4)$ & \\
\hline Female & $2,931 / 9,826(29.8)$ & $2,329 / 7,860(29.6)$ & $602 / 1,966(30.6)$ & \\
\hline Race/ethnicity - no./total no. (\%) & & & & 0.190 \\
\hline Yellow & $158 / 9,806(1.6)$ & $128 / 7,845(1.6)$ & $30 / 1,961(1.5)$ & \\
\hline White & $7,718 / 9,806(78.7)$ & $6,206 / 7,845(79.1)$ & $1,512 / 1,961(77.1)$ & \\
\hline Brown & $1,506 / 9,806(15.3)$ & $1,183 / 7,845(15.1)$ & $323 / 1,961(16.5)$ & \\
\hline Black & $424 / 9,806(4.3)$ & $328 / 7,845(4.2)$ & $96 / 1,961(4.9)$ & \\
\hline Hypertension - no./total no. (\%) & $8,245 / 9,826(83.9)$ & $6,592 / 7,860(83.9)$ & $1,653 / 1,966(84.1)$ & 0.837 \\
\hline Diabetes - no./total no. $(\%)$ & $4,158 / 9,826(42.3)$ & $3,372 / 7,860(42.9)$ & $786 / 1,966(40.0)$ & 0.020 \\
\hline Family history of early CAD - no./total no. (\%) & $1,501 / 9,826(15.3)$ & $1,204 / 7,860(15.3)$ & $297 / 1,966(15.1)$ & 0.834 \\
\hline Smoking - no./total no. (\%) & & & & 0.485 \\
\hline Absent & $4,598 / 9,826(46.8)$ & $3,698 / 7,860(47.0)$ & $900 / 1,966(45.8)$ & \\
\hline Current & $1,962 / 9,826(20.0)$ & $1,553 / 7,860(19.8)$ & $409 / 1,966(20.8)$ & \\
\hline Previous & $3,266 / 9,826(33.2)$ & $2,609 / 7,860(33.2)$ & $657 / 1,966(33.4)$ & \\
\hline COPD - no./total no. (\%) & $386 / 9,826(3.9)$ & $313 / 7,860(4.0)$ & $73 / 1,966(3.7)$ & 0.650 \\
\hline Previous TIA - no./total no. (\%) & $96 / 9,826(1.0)$ & $80 / 7,860(1.0)$ & $16 / 1,966(0.8)$ & 0.521 \\
\hline Previous Stroke - no./total no. (\%) & $381 / 9,826(3.9)$ & $304 / 7,860(3.9)$ & $77 / 1,966(3.9)$ & 0.896 \\
\hline Creatinine $>1.5 \mathrm{mg} / \mathrm{dL}$ - no./total no. $(\%)$ & $835 / 9,826(8.5)$ & $687 / 7,860(8.7)$ & $148 / 1,966(7.5)$ & 0.086 \\
\hline Carotid obstruction > 50\% - no./total no. $(\%)$ & $511 / 9,826(5.2)$ & $425 / 7,860(5.4)$ & $86 / 1,966(4.4)$ & 0.069 \\
\hline
\end{tabular}


Research, Society and Development, v. 11, n. 2, e34211225828, 2022

(CC BY 4.0) | ISSN 2525-3409 | DOI: http://dx.doi.org/10.33448/rsd-v11i2.25828

PAD - no./total no. $(\%)$

$806 / 9,826(8.2) \quad 641 / 7,860(8.2)$

$165 / 1,966(8.4)$

0.748

Total cholesterol > $200 \mathrm{mg} / \mathrm{dL}$ - no./total no. $(\%)$

$5,218 / 9,826(53.1) \quad 4,170 / 7,860(53.1)$

$1,048 / 1,966(53.3) \quad 0.860$

Triglycerides $>200 \mathrm{mg} / \mathrm{dL}$ - no./total no. $(\%)$

$1,669 / 9,826(17.0)$

$1,352 / 7,860(17.2)$

$317 / 1,966(16.1)$

0.268

Previous AMI - no./total no. (\%)

0.397

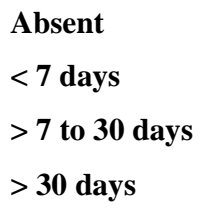

$\begin{array}{clc}4,875 / 9,796(49.8) & 3,917 / 7,839(50.0) & 958 / 1,957(49.0) \\ 367 / 9,796(3.7) & 283 / 7,839(3.6) & 84 / 1,957(4.3) \\ 939 / 9,796(9.6) & 759 / 7,839(9.7) & 180 / 1,957(9.2) \\ 3,615 / 9,796(36.9) & 2,880 / 7,839(36.7) & 735 / 1,957(37.6)\end{array}$

Dyspnea (NYHA) - no./total no. (\%)

Absent
I
III
IV
Angina (CCS) - no./total no. $(\%)$

$\begin{array}{rcr}4,848 / 9,826(49.3) & 3,903 / 7,860(49.7) & 945 / 1,966(48.1) \\ 2,794 / 9,826(28.4) & 2,213 / 7,860(28.2) & 581 / 1,966(29.6) \\ 1,712 / 9,826(17.4) & 1,366 / 7,860(17.4) & 346 / 1,966(17.6) \\ 432 / 9,826(4.4) & 343 / 7,860(4.4) & 89 / 1 ., 966(4.5) \\ 40 / 9,826(0.4) & 35 / 7,860(0.4) & 5 / 1,966(0.3)\end{array}$

0.508

Absent
II
III
IV

Clinical condition - no./total no. (\%)

$\begin{array}{ccc}3,153 / 9,826(32.1) & 2,548 / 7,860(32.4) & 605 / 1,966(30.8) \\ 798 / 9,826(8.1) & 624 / 7,860(7.9) & 174 / 1,966(8.9) \\ 3,256 / 9,826(33.1) & 2,619 / 7,860(33.3) & 637 / 1,966(32.4) \\ 1,318 / 9,826(13.4) & 1,032 / 7,860(13.1) & 286 / 1,966(14.5) \\ 1,301 / 9,826(13.2) & 1,037 / 7,860(13.2) & 264 / 1,966(13.4)\end{array}$

0.214

\section{Asymptomatic after AMI}

Stable angina

Unstable angina

Silent ischemia

Coronary involvement - no./total no. (\%) angiogram

LMD

One-vessel

Two-vessel

Three-vessel

LVEF \% - no./total no. (\%)

$\begin{array}{cccc}2,314 / 9,768(23.7) & 1,850 / 7,812(23.7) & 464 / 1,956(23.7) & \\ 5,084 / 9,768(52.0) & 4,051 / 7,812(51.9) & 1,033 / 1,956(52.8) & \\ 1,538 / 9,768(15.7) & 1,222 / 7,812(15.6) & 316 / 1,956(16.2) & \\ 832 / 9,768(8.5) & 689 / 7,812(8.8) & 143 / 1,956(7.3) & \\ & & & 0.266 \\ & & & \\ 2,102 / 9,796(21.4) & 1,663 / 7,835(21.2) & 439 / 1,961(22.4) & \\ 531 / 9,796(5.4) & 412 / 7,835(5.2) & 119 / 1,961(6.1) & \\ 2,083 / 9,796(21.3) & 1,693 / 7.835(21.6) & 390 / 1,961(19.9) & \\ 5,080 / 9,796(51.8) & 4,067 / 7,835(51.9) & 1,013 / 1,961(51.6) & \\ & & & 0.630\end{array}$


Research, Society and Development, v. 11, n. 2, e34211225828, 2022

(CC BY 4.0) | ISSN 2525-3409 | DOI: http://dx.doi.org/10.33448/rsd-v11i2.25828

\begin{tabular}{|c|c|c|c|c|}
\hline$\geq 55$ & $3,651 / 9,767$ (37.4) & $2,934 / 7,813(37.6)$ & $717 / 1,954$ (36.7) & \\
\hline$\geq 45$ to $<55$ & $2,824 / 9,767$ (28.9) & $2,238 / 7,813(28.6)$ & $586 / 1,954(30.0)$ & \\
\hline$\geq 30$ to $<45$ & $2,317 / 9,767$ (23.7) & $1,865 / 7,813$ (23.9) & $452 / 1,954(23.1)$ & \\
\hline$<30$ & 975/9,767 (10.0) & 776/7,813 (9.9) & $199 / 1,954(10.2)$ & \\
\hline Surgical procedure - no./total no. (\%) & & & & 0.539 \\
\hline Elective & 9,586/9,795 (97.9) & $7,668 / 7,836$ (97.9) & $1,918 / 1,959$ (97.9) & \\
\hline Urgent & $182 / 9,795$ (1.9) & $144 / 7,836(1.8)$ & $38 / 1,959(1.9)$ & \\
\hline Emergent & $27 / 9,795(0.3)$ & $24 / 7,836(0.3)$ & $3 / 1,959(0.2)$ & \\
\hline
\end{tabular}

CAD, coronary artery disease; COPD, chronic obstructive pulmonary disease; TIA, transient ischemic attack; PAD, peripheral arterial disease; AMI, acute myocardial infarction; NYHA, New York Heart Association; CCS, Canadian Cardiovascular Society; LMD, Left main disease; LVEF, left ventricle ejection fraction. Source: Authors.

Analyzing the perioperative data, there was also no statistical difference between the construction and validation groups, according to the analyzed criteria (Table 3 ). 
Research, Society and Development, v. 11, n. 2, e34211225828, 2022

(CC BY 4.0) | ISSN 2525-3409 | DOI: http://dx.doi.org/10.33448/rsd-v11i2.25828

Table 3. Intra and postoperative characteristics of the construction and validation groups.

\begin{tabular}{|c|c|c|c|c|}
\hline Characteristic & $\begin{array}{l}\text { Total } \\
(\mathrm{N}=\mathbf{9 , 8 2 6})\end{array}$ & $\begin{array}{l}\text { Construction } \\
(\mathbf{N}=\mathbf{7 , 8 6 0})\end{array}$ & $\begin{array}{l}\text { Validation } \\
(\mathrm{N}=\mathbf{1 , 9 6 6 )}\end{array}$ & P-Value \\
\hline Cardiopulmary bypass no/total no (\%) & & & & 0.321 \\
\hline Yes & $9,600 / 9,796(98,0)$ & $7,682 / 7,833(98.1)$ & $1,918 / 1,963(97,7)$ & \\
\hline No & $196 / 9,796(2.0)$ & $151 / 7,833(1.9)$ & $45 / 1.963(2.3)$ & \\
\hline Perfusion duration, min & & & & 0.559 \\
\hline Mean & $82.30 \pm 39.16 / 9,623$ & $82.19 \pm 37.47 / 7,704$ & $82.77 \pm 45.30 / 1,919$ & \\
\hline Median & $80.0 / 9,623$ & $80.0 / 7,704$ & $80.0 / 1,919$ & \\
\hline Anoxia duration, min & & & & 0.903 \\
\hline Mean & $54.98 \pm 18.79 / 9,601$ & $54.98 \pm 18.83 / 7,686$ & $54.97 \pm 18.63 / 1,915$ & \\
\hline Median & $54.0 / 9,601$ & $54.0 / 7,686$ & $54.0 / 1,915$ & \\
\hline $\mathbf{N}^{0}$ grafts performed & & & & 0.829 \\
\hline Mean & $2.72 / 9,826$ & $2.72 / 7,860$ & $2.72 / 1,966$ & \\
\hline Median & $3.0 / 9,826$ & $3.0 / 7,860$ & $3.0 / 1,966$ & \\
\hline Mammary artery graft no/total no (\%) & & & & 0.637 \\
\hline Yes & $9,064 / 9,826(92.2)$ & $7,245 / 7,860(92.2)$ & $1,819 / 1,966(92.5)$ & \\
\hline No & $762 / 9,826(7.8)$ & $615 / 7,860(7.8)$ & $147 / 1,966(7.5)$ & \\
\hline \multicolumn{5}{|l|}{ Hospitalization length after CABG, days } \\
\hline ICU & $3.38 \pm 7.52$ & $3.30 \pm 6.84$ & $3.70 \pm 9.76$ & 0.430 \\
\hline Mean & $9.76 \pm 17.54$ & $9.68 \pm 16.38$ & $10.05 \pm 21.55$ & 0.893 \\
\hline Median & 7.0 & 7.0 & 7.0 & 0.893 \\
\hline Outcome, no/total no (\%) & & & & 0.817 \\
\hline Hospital discharge & $9,337 / 9,826(95.0)$ & $7,471 / 7,860(95.1)$ & $1,866 / 1,966(94.9)$ & \\
\hline Death & $489 / 9,826(5.0)$ & $389 / 7,860(4.9)$ & $100 / 1,966(5.1)$ & \\
\hline
\end{tabular}

Min, minutes; $\mathrm{CABG}$, coronary artery bypass grafting; ICU, intensive unit care. Source: Authors.

\subsection{Univariate analysis: construction group}

Univariate analysis of patients sociodemographic and clinical characteristics identified ten factors before CABG (age, sex, hypertension, previous stroke, creatinine level $>1.5 \mathrm{mg} / \mathrm{dL}$, carotid obstruction $>50 \%$, PAD, previous AMI, dyspnea, and angina) with statistical significance associated with the death outcome. Factor, such as ethnicity, diabetes, family history of early CAD, smoking, chronic obstructive pulmonary disease (COPD), transient ischemic attack (TIA), and dyslipidemia, did not show statistically significant associations with the death outcomes. (Table 4). 
Research, Society and Development, v. 11, n. 2, e34211225828, 2022

(CC BY 4.0) | ISSN 2525-3409 | DOI: http://dx.doi.org/10.33448/rsd-v11i2.25828

Table 4. Univariate analysis: construction group: previous characteristic before CABG and in-hospital death after surgery.

\begin{tabular}{|c|c|c|c|}
\hline Characteristic & $\begin{array}{l}\text { Total } \\
(\mathrm{N}=7.860)\end{array}$ & Mortality & P-Value \\
\hline Age in years - no./total no. (\%) & & & $<0,001$ \\
\hline$\leq \mathbf{5 0}$ & $890 / 7.850(11,3)$ & $20 / 890(2,2)$ & \\
\hline $50-69$ & $5.003 / 7.850(63,7)$ & $176 / 5.003(3,5)$ & \\
\hline$\geq 70$ & $1.957 / 7.850(24,7)$ & $191 / 1.957(9,8)$ & \\
\hline Sex - no./total no. $(\%)$ & & & $<0,001$ \\
\hline Male & $5.531 / 7.860(70,4)$ & $219 / 5.531(4,0)$ & \\
\hline Female & $2.329 / 7.860(29,6)$ & $170 / 2.329(7,3)$ & \\
\hline Race/ethnicity - no./total no. (\%) & & & 0,515 \\
\hline Yellow & $128 / 7.845(1,6)$ & $10 / 128(7,8)$ & \\
\hline White & $6.206 / 7.845(79,0)$ & $303 / 6.206(4,9)$ & \\
\hline Brown & $1.183 / 7.845(15,1)$ & $57 / 1.183(4,8)$ & \\
\hline Black & $328 / 7.845(4,2)$ & $19 / 328(5,8)$ & \\
\hline Hypertension - no./total no. (\%) & $6.592 / 7.860(83,9)$ & $345 / 6.592(5,2)$ & 0,007 \\
\hline Diabetes - no./total no. $(\%)$ & $3.372 / 7.860(42,9)$ & $182 / 3.372(5,4)$ & 0,115 \\
\hline Family history of early CAD - no./total no. (\%) & $1.204 / 7.860(15,3)$ & $59 / 1.204(4,9)$ & 1,00 \\
\hline Smoking - no./total no. (\%) & & & 0,315 \\
\hline Absent & $3.698 / 7.860(47)$ & $197 / 3.698(5,3)$ & \\
\hline Current & $1.553 / 7.860(19,8)$ & $75 / 1.553(4,8)$ & \\
\hline Previous & $2.609 / 7.860(33,2)$ & $117 / 2.609(4,5)$ & \\
\hline COPD - no./total no. $(\%)$ & $313 / 7.860(4,0)$ & $21 / 313(6,7)$ & 0,143 \\
\hline Previous TIA - no./total no. (\%) & $80 / 7.860(1,0)$ & $7 / 80(8,8)$ & 0,117 \\
\hline Previous Stroke - no./total no. (\%) & $304 / 7.860(3,9)$ & $26 / 304(8,6)$ & 0,006 \\
\hline Creatinine > 1.5 mg/dL - no./total no. $(\%)$ & $687 / 7.860(8,7)$ & $72 / 687(10,5)$ & $<0,001$ \\
\hline Carotid obstruction > 50\% - no./total no. $(\%)$ & $425 / 7.860(5,4)$ & $46 / 425(10,8)$ & $<0,001$ \\
\hline PAD - no./total no. (\%) & $641 / 7.860(8,2)$ & $54 / 641(8,4)$ & $<0,001$ \\
\hline Total cholesterol > $200 \mathrm{mg} / \mathrm{dL}$ - no./total no. $(\%)$ & $4.170 / 7.860(53,1)$ & $207 / 4.170(5,0)$ & 0,958 \\
\hline Triglycerides > 200 mg/dL - no./total no. $(\%)$ & $1.352 / 7.860(17,2)$ & $58 / 1.352(4,3)$ & 0,241 \\
\hline Previous AMI - no./total no. (\%) & & & $<0,001$ \\
\hline Absent & $3.917 / 7.839(50,0)$ & $184 / 3.917(4,7)$ & \\
\hline$<7$ days & $283 / 7.839(3,6)$ & $42 / 283(14,8)$ & \\
\hline$>7$ to 30 days & $759 / 7.839(9,7)$ & $51 / 759(6,7)$ & \\
\hline$>30$ days & $2.880 / 7.839(36,7)$ & $111 / 2.880(3,9)$ & \\
\hline Clinical condition - no./total no. (\%) & & & $<0,001$ \\
\hline Asymptomatic after AMI & $1.850 / 7.812(23,7)$ & $102 / 1.850(5,5)$ & \\
\hline Stable angina & $4.051 / 7.812(51,9)$ & $164 / 4.051(4,0)$ & \\
\hline Unstable angina & $1.222 / 7.812(15,6)$ & $88 / 1.222(7,2)$ & \\
\hline Silent ischemia & $689 / 7.812(8,8)$ & $32 / 689(4,6)$ & \\
\hline
\end{tabular}


Research, Society and Development, v. 11, n. 2, e34211225828, 2022

(CC BY 4.0) | ISSN 2525-3409 | DOI: http://dx.doi.org/10.33448/rsd-v11i2.25828

\begin{tabular}{|c|c|c|c|}
\hline Dyspnea (NYHA) - no./total no. (\%) & & & $<0,001$ \\
\hline Absent & $3.903 / 7.860(49,7)$ & $201 / 3.903(5,1)$ & \\
\hline $\mathbf{I}$ & $2.213 / 7.860(28,2)$ & $84 / 2.213(3,8)$ & \\
\hline II & $1.366 / 7.860(17,4)$ & $63 / 1.366(4,6)$ & \\
\hline III & $343 / 7.860(4,4)$ & $35 / 343(10,2)$ & \\
\hline IV & $35 / 7.860(0,4)$ & $6 / 35(17,1)$ & \\
\hline Angina (CCS) - no./total no. (\%) & & & $<0,001$ \\
\hline Absent & $2.548 / 7.860(32,4)$ & $136 / 2.548(5,3)$ & \\
\hline $\mathbf{I}$ & $624 / 7.860(7,9)$ & $20 / 624(3,2)$ & \\
\hline II & $2.619 / 7.860(33,3)$ & $96 / 2.619(3,7)$ & \\
\hline III & $1.032 / 7.860(13,1)$ & $53 / 1.032(5,1)$ & \\
\hline IV & $1.037 / 7.860(13,2)$ & $84 / 1.037(8,1)$ & \\
\hline LMD - no./total no. (\%) & $1.663 / 7.835(21,2)$ & $117 / 1.663(7,0)$ & $<0,001$ \\
\hline LVEF, \% - no./total no. (\%) & & & $<0,001$ \\
\hline$\geq 55$ & $2.934 / 7.813(37,6)$ & $122 / 2.934(4,2)$ & \\
\hline$\geq 45$ to $<55$ & $2.238 / 7.813(28,6)$ & $89 / 2.238(4,0)$ & \\
\hline$\geq 30$ to $<45$ & $1.865 / 7.813(23,9)$ & $116 / 1.865(6,2)$ & \\
\hline$<30$ & $776 / 7.813(9,9)$ & $58 / 776(7,5)$ & \\
\hline
\end{tabular}

CAD, coronary artery disease; COPD, chronic obstructive pulmonary disease; TIA, transient ischemic attack; PAD, peripheral arterial disease; AMI, acute myocardial infarction; NYHA, New York Heart Association; CCS, Canadian Cardiovascular Society; LMD, Left main disease; LVEF, left ventricle ejection fraction. Source: Authors.

\subsection{Multivariate analysis and preoperative risk score}

Antecedents, such as systemic arterial hypertension, carotid obstruction $>50 \%$, and PAD, found to increase the risk of hospital death in the univariate analysis, presented a neutral association with the outcome in the multivariate analysis. As in the univariate analysis, diabetes did not show an increase in the risk of in-hospital death. In contrast, age $\geq 70$ years and emergency CABG had the strongest association with in-hospital death.

The time elapsed between AMI and surgery was inversely proportionate, i.e., the more recent the AMI, the greater the risk of in-hospital death in the postoperative period.

Noteworthy injury of LMD, represented by stenosis $\geq 50 \%$ on coronary angiography, proved to be an independent risk factor for postoperative in-hospital death.

After multivariate analysis, it was possible to identify the factors that influenced outcomes. Fifteen (age, sex, hypertension, diabetes, previous neurological event, creatinine level $>1.5 \mathrm{mg} / \mathrm{dL}$, carotid obstruction $>50 \%$, peripheral arterial disease, previous acute myocardial, infarction, left ventricular ejection fraction $<45 \%$, dyspnea class III and IV by NYHA, angina class IV by CCS, urgent and emergent surgical procedure, left main disease $>50 \%$ and current smoker) variables were selected according to their statistical association with death or clinical importance, according to current data in the literature, and a nomogram was constructed (Figure 1). 
Research, Society and Development, v. 11, n. 2, e34211225828, 2022

(CC BY 4.0) | ISSN 2525-3409 | DOI: http://dx.doi.org/10.33448/rsd-v11i2.25828

Figure 1. ERPO risk score nomogram.

Points
Age
Sex
Hypertension
Diabetes
PNE
Creatinine $>1.5 \mathrm{mg} / \mathrm{dl}$
CAD $>50 \%$
PAD
Previous AMI
EF $<45 \%$
Dyspnea NYHA III or IV
Angina CCS 4
Urg. or Emerg. Surgery
LMD
Current smoking
Total points
Linear predictors

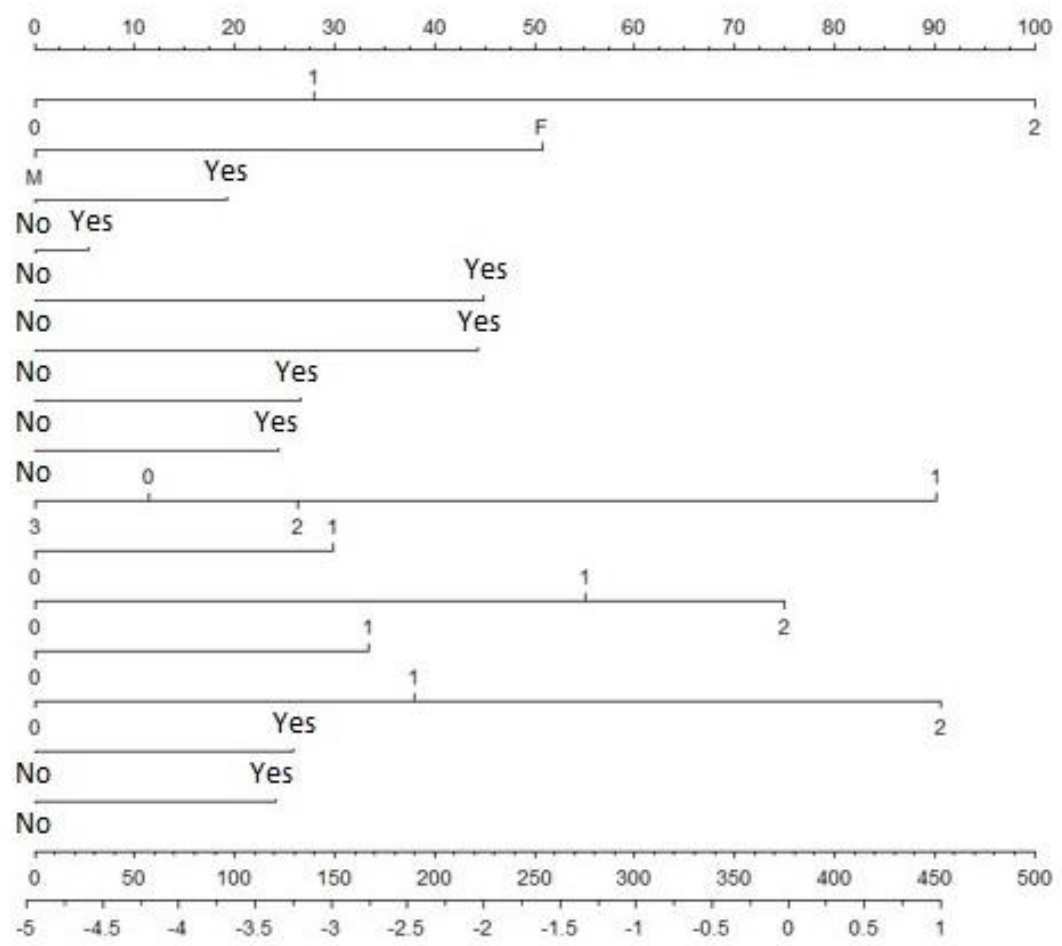

PNE, previous neurological event; CAD, coronary artery disease; PAD, peripheral arterial disease; AMI, acute myocardial infarction; EF, ejection fraction; NYHA, New York Heart Association; CCS, Canadian Cardiovascular Society; Urg or Emerg., urgent or emergent; LMD, Left main disease. Source: Authors.

The total number of points obtained in each of the 15 variables included in the model ranged from 0 to 500 . Age was the coefficient with the highest possible score, reaching 100 points when the individual was $\geq 70$ years. Other risk factors showed scores ranging from 5 (diabetes) to 91 (emergency surgery) (Table 5). 
Research, Society and Development, v. 11, n. 2, e34211225828, 2022

(CC BY 4.0) | ISSN 2525-3409 | DOI: http://dx.doi.org/10.33448/rsd-v11i2.25828

Table 5. In-hospital death risk through multivariate regression and the developed preoperative risk score (PRS).

\begin{tabular}{|c|c|c|c|c|}
\hline Variable & Regression coefficient & P-value & OR $(95 \% \mathrm{CI})$ & Score points \\
\hline \multicolumn{5}{|l|}{ Age (years) } \\
\hline$\leq 50$ & & $<0.001$ & 1 & 0 \\
\hline $50-69$ & 0.367 & 0.134 & $1.443(0.893-2.332)$ & 28 \\
\hline$\geq 70$ & 1.313 & $<0.001$ & $3.716(2.272-6.077)$ & 100 \\
\hline \multicolumn{5}{|l|}{ Sex } \\
\hline Male & & & 1 & 0 \\
\hline Female & 0.667 & $<0.001$ & $1.948(1.560-2.433)$ & 51 \\
\hline Hypertension & 0.253 & 0.143 & $1.288(0.918-1.807)$ & 19 \\
\hline Diabetes & 0.071 & 0.528 & $1.073(0.862-1.337)$ & 5 \\
\hline PNE & 0.590 & 0.003 & $1.804(1.216--2.677)$ & 45 \\
\hline Creatinine level $>1.5 \mathrm{mg} / \mathrm{dL}$ & 0.581 & $<0.001$ & $1.787(1.328-2.406)$ & 44 \\
\hline Carotid obstruction $>50 \%$ & 0.349 & 0.055 & $1.418(0.992-2.026)$ & 27 \\
\hline PAD & 0.320 & 0.057 & $1.377(0.990-1.915)$ & 24 \\
\hline \multicolumn{5}{|l|}{ Previous AMI } \\
\hline Absent & & $<0.001$ & 1 & 11 \\
\hline$<7$ days & 1.034 & $<0.001$ & $2.811(1.892--4.176)$ & 90 \\
\hline$>7$ to 30 days & 0.196 & 0.267 & $1.216(0.861-1.719)$ & 26 \\
\hline$>30$ days & -0.150 & 0.252 & $0.861(0.665-1.113)$ & 0 \\
\hline LVEF $<45 \%$ & 0.393 & 0.001 & $1.481(1.180-1.858)$ & 30 \\
\hline \multicolumn{5}{|l|}{ Dyspnea (NYHA) } \\
\hline III & 0.724 & $<0.001$ & $2.062(1.399-3.038)$ & 55 \\
\hline IV & 0.984 & 0.046 & $2.674(1.016-7.037)$ & 75 \\
\hline $\operatorname{CCS} 4$ & 0.438 & 0.001 & $1.550(1.186-2.025)$ & 33 \\
\hline \multicolumn{5}{|l|}{ Surgical procedure } \\
\hline Elective & & 0.024 & 1 & 0 \\
\hline Urgent & 0.498 & 0.080 & $1.646(0.941-2.877)$ & 38 \\
\hline Emergent & 1.190 & 0.028 & $3.288(1.138-9.505)$ & 91 \\
\hline LMD $>50 \%$ & 0.339 & 0.005 & $1.404(1.106-1.782)$ & 26 \\
\hline Current smoker & 0.317 & 0.029 & $1.373(1.034-1.823)$ & 24 \\
\hline Constant & -4.130 & $<0.001$ & & \\
\hline
\end{tabular}

PNE, previous neurological event; PAD, peripheral arterial disease; AMI, acute myocardial infarction; LVEF, left ventricular ejection fraction; NYHA, New York Heart Association; CCS, Canadian Cardiovascular Society; LMD, Left main disease. Source: Authors.

The final logistic model was tested in the construction group, and a C-statistic of 0.745 (95\% CI, $0.720-0.770$ ) indicated a satisfactory discriminatory power. Score discrimination was tested by measuring the area under the receiver operating characteristic (ROC) curve of the validation group, which was 0.716 (95\% CI, 0.666-0.767), (Figure 2). 
Research, Society and Development, v. 11, n. 2, e34211225828, 2022

(CC BY 4.0) | ISSN 2525-3409 | DOI: http://dx.doi.org/10.33448/rsd-v11i2.25828

Figure 2. Area under the ROC curve for risk models applied to construction and validation group.

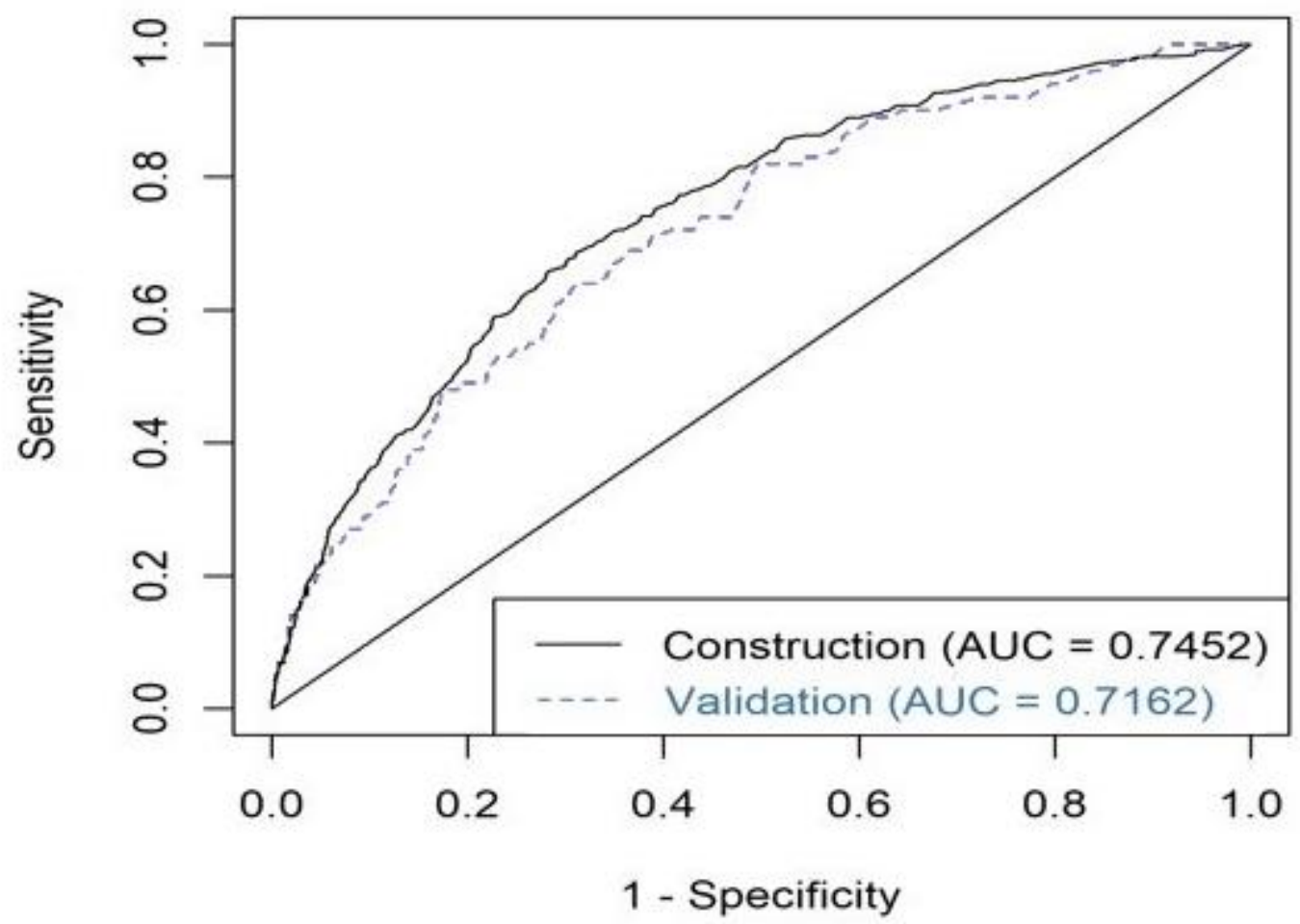

*In the construction group, the area under the ROC curve was 0.745 (95\% CI, 0.720-0.770). Score discrimination applied to 1,966 patients in the validation group identified an area under ROC of 0.716 (95\% CI, 0.666-0.767). Source: Authors.

Based on the total points accumulated from each item of the multivariate analysis, patients were divided into four groups: low risk ( $\leq 84$ points) (30\%), intermediate risk (85-152 points) (40\%), high risk (153-211 points) (20\%), and very high risk (>211 points) (10\%) (Figure 3). The risk of death ranged from 1.2\% in those classified as low risk, 3.3\% in the intermediate group, $7.9 \%$ in the high risk and $16.3 \%$ in the very high-risk group. 
Figure 3. Risk of death stratification according to a preoperative risk score (PRS).
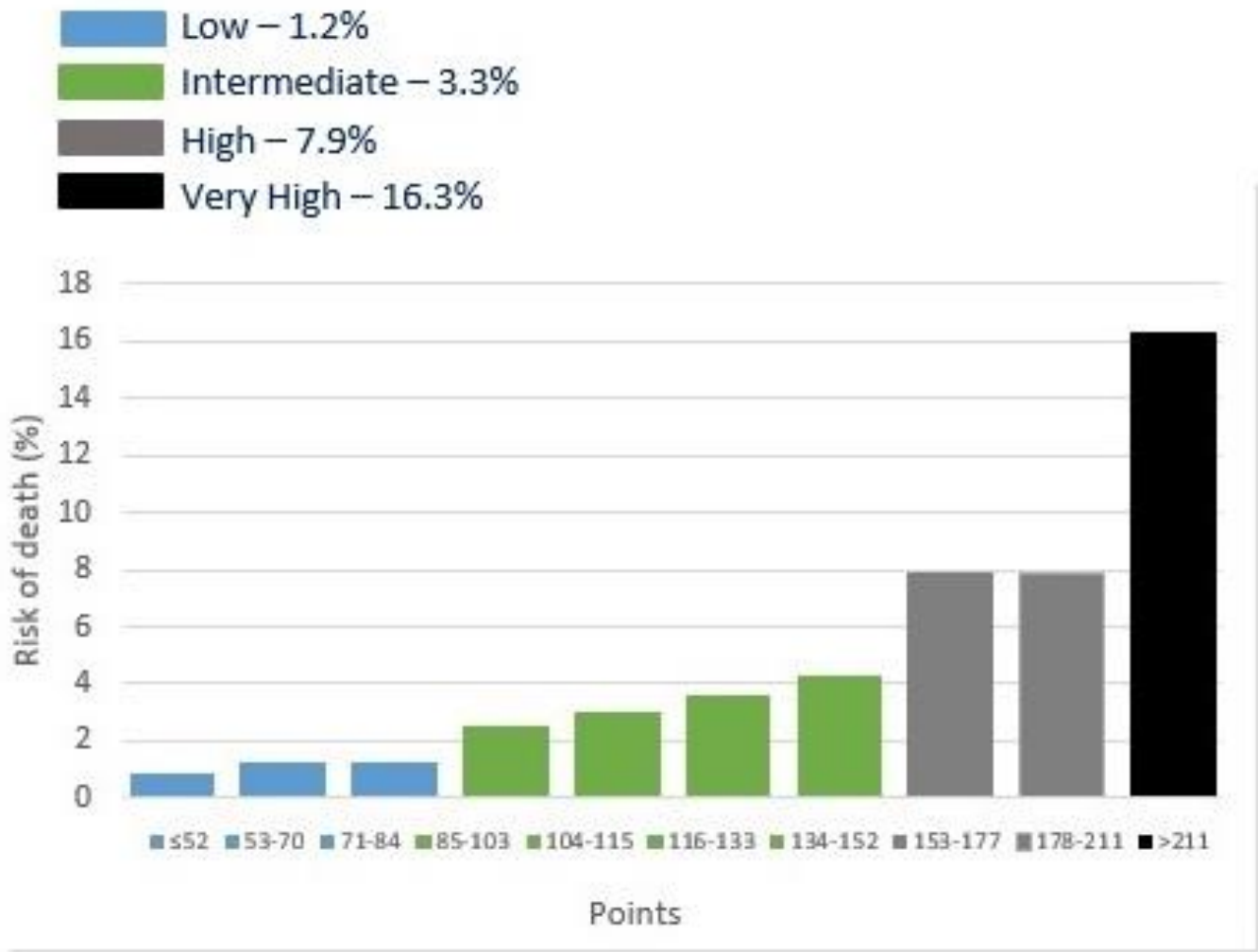

*Low risk (score $\leq 84$ points) representing $30 \%$ of the sample, intermediate risk (score between $85-152$ points) consisting of $40 \%$ of everyone, high risk (score between 153-211) composed of $20 \%$ of patients and very high risk high (score> 211 points) formed by $10 \%$ of the sample. After the sum of the points and classification between one of the four categories, the average risk of death among patients considered low, intermediate, high and very high risk of death was 1,2\%, 3,3\%, 7,9\% and 16,3\%, respectively. Source: Authors.

\section{Discussion}

\subsection{Demographic Aspects}

The present study revealed that the average age of patients undergoing CABG is 62.43 years. Patients were predominantly $<70$ years of age $(75.3 \%)$. However, the $\geq 70$-years subgroup had a higher rate of deaths $(9.7 \%$ vs $3.4 \%$; $\mathrm{P}$ $<0.001)$.

Mortality was more strongly associated with female sex (7.0\% in female participants vs. $4.1 \%$ in male participants; $\mathrm{P}<0.001$ ). A similar association was described in a review of 14 studies, where it was identified that after CABG, women had a higher risk of death, as much as twofold in some studies (Blasberg et al., 2011). Death from CAD in female patients was delayed by 10 years, on an average, compared with male patients. In this study, the protective effects of estrogen were no longer present in the vast majority of female patients. This association was also described in the EuroSCORE II study, where women had higher mortality than men after CABG (Nashef et al., 2012).

The predominant ethnic group was Caucasian (78.5\%). Ethnic differences were not characterized as a factor influencing mortality $(\mathrm{P}=0.329)$. 


\subsection{Previous comorbidities and symptoms}

Comorbidities with the highest prevalence in patients undergoing CABG were, in decreasing order, systemic arterial hypertension, dyslipidemia (total cholesterol level $>200 \mathrm{mg} / \mathrm{dL}$ and/or triglyceride level $>200 \mathrm{mg} / \mathrm{dL}$ ), smoking and previous AMI.

The univariate analysis showed that antecedents, such as hypertension, stroke, creatinine level $>1.5 \mathrm{mg} / \mathrm{dL}$, carotid obstruction $>50 \%$, PAD, previous AMI, and heart failure with reduced EF had a strong association with the death outcome. In contrast, history relevant to the development of $\mathrm{CAD}$, or even the progression of the speed of atherosclerotic disease, such as diabetes, a family history of early CAD, COPD, previous TIA, and dyslipidemia (total cholesterol level $>200 \mathrm{mg} / \mathrm{dL}$ and/or triglyceride level $>200 \mathrm{mg} / \mathrm{dL}$ ), showed a neutral association with death in the univariate analysis.

The mortality of patients with heart failure is directly proportional to the NYHA dyspnea class. Our results corroborated this concept, with the death outcome identified among 3.8\% of those with class I, $4.6 \%$ of those with class II, $10.2 \%$ of those with class III, and $17.1 \%$ of those with class IV dyspnea. Multivariate analysis showed dyspnea NYHA IV was the fourth largest factor implicated in a death, followed by $\geq 70$ years of age, emergency CABG, and AMI $<7$ days prior. Dyspnea and respective NYHA classes have also been described as factors with a strong correlation to the death outcomes according to the Chinese risk model for patients undergoing CABG (Hu et al., 2020). Dyspnea has the second most significant association with death, preceded only by previous cardiac surgery (Hu et al., 2020).

Among those who had angina before the procedure, the presence of angina at rest (CCS class IV) conferred a greater risk of death. In contrast, there was no association with outcome in those who presented angina only during strenuous, medium, and small efforts (CCS classes I, II, and III, respectively). A similar finding was described in EuroSCORE II (Nashef et al., 2012).

The analysis of clinical conditions prior to the surgical procedure revealed that those who presented with acute coronary syndrome (unstable angina or AMI), even if they had evolved asymptomatically afterward, had a higher risk of death. Patients not displaying acute coronary syndrome or who were asymptomatic (stable angina or who were indicated for surgery for silent ischemia) did not present an increased risk of death in the univariate analysis.

\subsection{Preoperative coronary angiography and left ventricular function analysis}

The association between anatomical injuries and death was also investigated. No direct association was found between the number of diseased arteries (one-vessel, two-vessel, or three-vessel) and death. However, patients who had LMD had a higher risk of death. When the risk of death in patients with LMD was compared with those with three-vessel disease, patients with LMD injury had a $48 \%$ higher risk of death than patients with three-vessel disease. Left ventricular function, analyzed under ventriculography in the catheterization laboratory, also showed a link with death. LVEF $\geq 45 \%$, considered as normal left ventricular function or mild EF reduction, was not a risk factor for death. However, LVEF < 45\%, classified as moderate to severe reduction of EF, proved to be an independent and directly proportional risk factor for death in the postoperative period. Therefore, those who had a severe reduction of EF (LVEF $<30 \%)$ were 1.19 times more at risk of death after CABG than those who had a moderate reduction of EF (LVEF 30-44\%). EuroSCORE II also identified that lower LVEF increased the risk of death in the postoperative period. In that risk score, individuals who had an LVEF <30\% had a 77\% higher risk of death than those who had an LVEF of 30-50\% (Nashef et al., 2012). The Chinese risk score also found that 
patients who had an LVEF <35\% had a risk of death 1.76 times higher than those who had an LVEF of 35-45\% (Hu et al., 2020).

\subsection{Surgical Procedure}

The surgical analysis showed that urgent or emergent surgeries performed non-electively had a higher risk of death than elective surgeries, most likely because they were performed on patients with more severe status.

Regarding the use of grafts with the left internal mammary artery (LIMA), patients who underwent grafts without using the LIMA, despite the reason, had a higher risk of death than those who used them ( $\mathrm{P}<0.001)$, proving the protective role of LIMA. Thus, it was found that patients, who received LIMA as a graft -whether as a single graft or part of the grafts - had lower postoperative mortality, highlighting the value of using this conduit in CABG. A similar association was identified by Leavitt et al. (2001) who compared in-hospital mortality rates among 280 individuals undergoing CABG with and without the use of LIMA and found them to be $2.2 \%$ and $2814.9 \%$ (P <0.001), respectively. The use of LIMA was not included in the final risk model because the model aimed to assess the risk of postoperative death based on comorbidities

and preoperative factors. Similarly, the use of cardiopulmonary bypass, as portrayed by Hueb et al. (2010) showed no association with death compared with patients who underwent surgery without cardiopulmonary 286 bypass $(\mathrm{P}=0.180)$.

\subsection{Influence of preoperative factors and their relation rate with outcome through the preoperative risk score}

Preoperative risk stratification is an important tool for patients and physicians to define an optimal therapeutic regimen, and for future research to identify the risk of CABG for individual patients. Age was shown to be the factor with the greatest possible impact on the PRS analysis outcome. Of the 500 points possible in the analysis, age corresponded with up to 100 points $(20 \%)$.

Neurological vascular disease, representing the sum of TIA and stroke, was associated with a higher risk of death in the PRS analysis. The association between a PNE and death was due to stroke because a previous TIA had a neutral association with death.

The presence of hypertension, diabetes, carotid obstruction $>50 \%$, and PAD had a low association with the mortality outcome. These factors were maintained in the final risk model due to their pathophysiological importance and analogy in the context of atherosclerotic disease.

Compared with the EuroSCORE II, the STS risk model, the New York CABG surgery risk score, and the new Chinese risk score with areas under the ROC curve of 0.80, 0.80, 0.78, and 0.78, respectively (D'Agostino et al., 2019; Hannan et al., 2013; Hu et al., 2020; Nashef et al., 2012), the PRS showed lower accuracy with an area under the ROC curve of 0.716. We believe that the comparison between the scores mentioned above and the PRS should be considered; however, we also note that (1) EuroSCORE II predicts death in cardiac surgery as a whole and may even combine procedures, such as CABG and valve approach (Nashef et al., 2012). Although it included four Brazilian centers, the score was based on a wide range of nations with a predominance of European centers (Nashef et al., 2012). (2) The STS risk model was based mostly on the North American and Canadian populations (D'Agostino et al., 2019). (3) The New York CABG surgery risk score was based on the population of New York (Hannan et al., 2013), and it does not include some factors associated with death in the postoperative period that are included in the STS, EuroSCORE II, and new Chinese risk score (D'Agostino et al., 2019; Hu 
et al., 2020; Nashef et al., 2012). Moreover, the inclusion of seven variables (versus 65 in STS, 18 in EuroScore II, and 16 in the Chinese risk score) may overlook factors associated with outcome (D’Agostino et al., 2019; Hu et al., 2020; Nashef et al., 2012). (4) Development of the new Chinese risk score included only centers located in China. Thus, it is not possible to affirm that the data and results can be extrapolated to the population of the West, including Brazil (Hu et al., 2020). The incidence of death in the construction group of our study was $4.9 \%$. We believe that this incidence is closer to the Brazilian reality compared with the rates described by STS (1.8\%), EuroScore II (3.9\%), and the Chinese risk model (2.1\%) (D’Agostino et al., 2019; Hu et al., 2020; Nashef et al., 2012).

PRS was created with the expectation of being able to estimate the risk of death in the postoperative hospital phase of $\mathrm{CABG}$, through inherent risk factors and the analysis of variables based on the Brazilian population in the preoperative period. The final score was predictive of death among patients undergoing isolated CABG with $71.6 \%$ accuracy in the validation group data. The model's good discriminatory power and subsequent validation of the score suggests that the identified variables are risk factors for postoperative mortality in the in-hospital phase. Additional validation of scores in other populations is required.

\subsection{Limitations}

The limitations of this study must be acknowledged. First, because this was a retrospective study with database analysis, some variables were under the effect of information bias. Second, there were no records in our database after hospital discharge; therefore, we could not measure the rate of early deaths after discharge (death within 30 days of surgery). Third, we could not calculate creatinine clearance because the data collection form did not specifically request the creatinine value. Fourth, there was no distinction between diabetic individuals who were orally treated and those who were insulindependent. This factor may have contributed to the fact that diabetes did not figure as a risk factor for death in the postoperative period.

\section{Conclusion}

PRS was shown to have a good power of distinction in defining patients of low, intermediate, high, and very high risk of death in the postoperative period following isolated CABG. The analysis of 15 relevant preoperative factors demonstrated its benefit as an auxiliary tool in preoperative preparation for surgical teams.

\section{Acknowledgments}

Jorge Alcantara Farran, MD; Silmara Cristina Friolani, MD; Carolina de Paulo Maldi, MD. Contributed to data collection

\section{References}

Blasberg, J. D., Schwartz, G. S., \& Balaram, S. K. (2011). The role of gender in coronary surgery. European Journal of Cardio-Thoracic Surgery, 40(3), 715-721. https://doi.org/10.1016/j.ejcts.2011.01.003

D’Agostino, R. S., Jacobs, J. P., Badhwar, V., Fernandez, F. G., Paone, G., Wormuth, D. W., \& Shahian, D. M. (2019). The Society of Thoracic Surgeons Adult Cardiac Surgery Database: 2019 Update on Outcomes and Quality. The Annals of Thoracic Surgery, 107(1), 24-32. 
Research, Society and Development, v. 11, n. 2, e34211225828, 2022

(CC BY 4.0) | ISSN 2525-3409 | DOI: http://dx.doi.org/10.33448/rsd-v11i2.25828

https://doi.org/10.1016/j.athoracsur.2018.10.004

Hannan, E. L., Farrell, L. S., Wechsler, A., Jordan, D., Lahey, S. J., Culliford, A. T., Gold, J. P., Higgins, R. S. D., \& Smith, C. R. (2013). The New York Risk Score for In-Hospital and 30-Day Mortality for Coronary Artery Bypass Graft Surgery. The Annals of Thoracic Surgery, 95(1), 46-52. https://doi.org/10.1016/j.athoracsur.2012.08.047

Health Ministery. (2021). DATASUS. (SUS informatics department). http://tabnet.datasus.gov.br.

Hu, Z., Chen, S., Du, J., Gu, D., Wang, Y., Hu, S., \& Zheng, Z. (2020). An In-hospital Mortality Risk Model for Patients Undergoing Coronary Artery Bypass Grafting in China. The Annals of Thoracic Surgery, 109(4), 1234-1242. https://doi.org/10.1016/j.athoracsur.2019.08.020

Hueb, W., Lopes, N. H., Pereira, A. C., Hueb, A. C., Soares, P. R., Favarato, D., D’Oliveira Vieira, R., Lima, E. G., Garzillo, C. L., da Silva Paulitch, F., Cesar, L. A. M., Gersh, B. J., \& Ramires, J. A. F. (2010). Five-Year Follow-Up of a Randomized Comparison Between Off-Pump and On-Pump Stable Multivessel Coronary Artery Bypass Grafting. The MASS III Trial. Circulation, 122(11_suppl_1), S48-S52. https://doi.org/10.1161/CIRCULATIONAHA.109.924258

Leavitt, B. J., O’Connor, G. T., Olmstead, E. M., Morton, J. R., Maloney, C. T., Dacey, L. J., Hernandez, F., \& Lahey, S. J. (2001). Use of the Internal Mammary Artery Graft and In-Hospital Mortality and Other Adverse Outcomes Associated With Coronary Artery Bypass Surgery. Circulation, 103(4), 507-512. https://doi.org/10.1161/01.CIR.103.4.507

Nashef, S. A. M., Roques, F., Sharples, L. D., Nilsson, J., Smith, C., Goldstone, A. R., \& Lockowandt, U. (2012). EuroSCORE II. European Journal of Cardio-Thoracic Surgery, 41(4), 734-745. https://doi.org/10.1093/ejcts/ezs043

Nilsson, J., Algotsson, L., Höglund, P., Lührs, C., \& Brandt, J. (2006). Comparison of 19 pre-operative risk stratification models in open-heart surgery. European Heart Journal, 27(7), 867-874. https://doi.org/10.1093/eurheartj/ehi720 